\title{
Limit laws for power sums and norms of i.i.d. samples
}

\author{
Anja Janßen
}

Received: 19 February 2008 / Revised: 3 December 2008 / Published online: 7 January 2009

(C) The Author(s) 2009. This article is published with open access at Springerlink.com

\begin{abstract}
We study the limit behavior of power sums and norms of i.i.d. positive samples from the max domain of attraction of an extreme value distribution. To this end, we combine limit theorems for sums and for maxima and use a link between extreme value theory and the Lévy measures of certain infinitely divisible laws, which are limit distributions of power sums. In connection with the von Mises representation of the Gumbel max domain of attraction, this new approach allows us to extend the limit results for power sums found in Ben Arous et al. (Probab Theory Relat Fields 132:579-612, 2005) and Bogachev (J Theor Probab 19:849-873, 2006). Furthermore, our findings shed a new light on the results of Schlather (Ann Probab 29:862-881, 2001) and treat the Gumbel case which is missing there.
\end{abstract}

Keywords Central limit theorem - Extreme value theory - Infinitely divisible distributions $\cdot l_{p}$-norms $\cdot$ Power sums $\cdot$ Stable distributions $\cdot$ von Mises representation

Mathematics Subject Classification (2000) $\quad 60 \mathrm{~F} 05 \cdot 60 \mathrm{E} 07 \cdot 60 \mathrm{G} 70$

\section{Introduction}

The use of $l_{p}$-norms is a well-known way of measuring vectors in $\mathbb{R}^{n}$. In the following, we study $l_{p}$-norms of random samples, where $X_{1}, X_{2}, \ldots$ are i.i.d. positive random

The author has been financially supported by the Georg Lichtenberg programm "Applied Statistics \& Empirical Methods".

\footnotetext{
A. Janßen $(\varangle)$

Institut für Mathematische Stochastik, Georg-August-Universität Göttingen,

Goldschmidtstr. 7, 37077 Göttingen, Germany

e-mail: janssen@math.uni-goettingen.de
} 
variables. Let the $l_{p}$-norm $\|\cdot\|_{p}$ of a positive sample $X_{1 n}=\left(X_{1}, X_{2}, \ldots, X_{n}\right)$ be defined as

$$
\left\|X_{1 n}\right\|_{p}=\left(\sum_{i=1}^{n} X_{i}^{p}\right)^{1 / p}, \quad p>0
$$

and $\left\|X_{1 n}\right\|_{\infty}=\max \left\{X_{1}, \ldots, X_{n}\right\}$. For $p=1$ or $p=\infty$ and $n \rightarrow \infty$ the limit behavior of these norms is well explored in terms of limit results for sums and for maxima of i.i.d. samples. Here, however, we allow $p$ to grow with $n$, thus looking at $l_{p(n)}$-norms of samples as $n \rightarrow \infty$. Our analysis of $l_{p(n)}$-norms of samples is based on the closely related behavior of power sums of the form

$$
\left(\left\|X_{1 n}\right\|_{p(n)}\right)^{p(n)}=\sum_{i=1}^{n} X_{i}^{p(n)} .
$$

For both norms and power sums, the limit behavior is determined by the distribution of $X_{1}$ and the behaviour of $p(n)$ as $n \rightarrow \infty$. Typically, an important role will be played by the largest summand, especially for heavy-tailed distributions of $X_{1}$ and fast-growing sequences $p(n)$. We show that nontrivial limit laws emerge if $p(n)$ is chosen in accordance with the tail behavior of $X_{1}$.

For samples $X_{1 n}$ of positive i.i.d. random variables and properly chosen sequences $p(n) \rightarrow \infty$ the limit behavior of the appropriately normalized power sums

$$
\frac{\sum_{i=1}^{n} X_{i}^{p(n)}-\hat{b}(n)}{\hat{a}(n)}
$$

has been studied recently in [1] and [2]. Both articles are based on a Tauberian approach and assume a certain asymptotic tail behavior of $X_{1}$. Surprisingly, the emerging families of limit distributions are identical for the different tail behaviors studied there. We will demonstrate that this generality is no coincidence, that is, we will show that the limit behavior of power sums and norms of samples is basically governed by max domains of attraction. Now, since the distributions studied in [1] and [2] belong to the Gumbel max domain of attraction, they yield the same family of limit laws.

While it is illustrated in [1] that the analysis of power sums has applications, for example, in branching processes and the Random Energy Model (see [3]), the limit distributions of norms are of theoretical interest, since they build a smooth transition between limit laws for sums and for maxima.

For samples $X_{1 n}$ of i.i.d. positive random variables from the max domain of attraction of the Weibull or Fréchet distribution the limit laws for the suitably normalized $l_{p(n)}$-norms

$$
\frac{\left\|X_{1 n}\right\|_{p(n)}-\tilde{b}_{c}(n)}{\tilde{a}_{c}(n)}
$$


have been derived in [11]. Schlather's method, which is based on the asymptotic tail behavior of the distribution of $X_{1}$, works well for the cases studied there but fails for the Gumbel domain of attraction as it contains distributions with a wide range of different tail behavior.

Linking between extreme value theory and limit theorems for sums, we follow a different approach. This connection is applicable to each of the three max domains of attraction in the same way as demonstrated in Sect. 2. For the Gumbel case, by use of the von Mises representation of its max domain of attraction, we obtain convergence to the family of distributions found in [1] and [2], which is stated in Sect. 3, and proved in Sect. 4. However, in some cases this convergence is restricted to certain subsequences. Section 5 provides such an example as well as some further results for the Gumbel case. Section 6 is dedicated to the Weibull and the Fréchet case.

\section{Fundamentals}

Our approach is based on extreme value theory. Consequently, all distributions that are considered here are assumed to be in the max domain of attraction of an extreme value distribution.

Let $X_{1}, X_{2}, \ldots$ be i.i.d. with distribution function $F$. Then $F$ is said to be in the max domain of attraction of an extreme value distribution with distribution function $G$ if there exist norming constants $a(n)$ and $b(n)$ such that

$$
\lim _{n \rightarrow \infty} P\left(\frac{\max \left\{X_{1}, \ldots, X_{n}\right\}-b(n)}{a(n)} \leq x\right)=G(x)
$$

for all $x \in \mathbb{R}$. Then $G$ belongs to one of the three possible extreme value distributions (see, e.g., [10]), namely

- the Weibull distribution with parameter $\alpha>0$

$$
\Psi_{\alpha}(x)=\exp \left(-(-x)^{\alpha}\right), \quad x \leq 0,
$$

- the Fréchet distribution with parameter $\alpha>0$

$$
\Phi_{\alpha}(x)=\exp \left(-x^{-\alpha}\right), \quad x \geq 0
$$

- the Gumbel distribution

$$
\Lambda(x)=\exp \left(-e^{-x}\right), \quad x \in \mathbb{R}
$$

We write $F \in \mathcal{D}_{\infty}(G)$ if $F$ is in the max domain of attraction of $G$.

Both limit theorems for maxima and for sums can be formulated in such a way that part of the necessary and sufficient conditions depends on the behavior of the tails of the distribution functions. This connection between sums and maxima, which, to our knowledge, has been established first in [9], p. 315, will form the base of our analysis. 
One can show (see, e.g., [5], Proposition 3.3.2) that Eq. 2.1 is equivalent to

$$
\lim _{n \rightarrow \infty} n \bar{F}(a(n) x+b(n))=-\log (G(x)), \quad x \in \mathbb{R},
$$

where $\bar{F}(\cdot)$ denotes the tail distribution function $1-F(\cdot)$ of $X_{1}$.

On the other hand, for sums

$$
S_{n}=\xi_{n, 1}+\cdots+\xi_{n, k_{n}}-b(n)
$$

of independent and infinitesimal random variables $\xi_{n, l}$ with distribution functions $F_{n}$, $n \in \mathbb{N}, 1 \leq l \leq k_{n}$, a criterion for convergence as formulated in [7], pp. 116-117, includes an expression similar to (2.2). Here, it is necessary that there exist nondecreasing functions

$$
M(\cdot) \text { with } M(-\infty)=0 \text { and } N(\cdot) \text { with } N(+\infty)=0
$$

defined on $[-\infty, 0)$ and $(0,+\infty]$, respectively, such that at every continuity point of $M(u)$ and $N(u)$ it holds that

$$
\begin{aligned}
& \lim _{n \rightarrow \infty} k_{n} F_{n}(u)=M(u), \quad u<0, \\
& \lim _{n \rightarrow \infty} k_{n} \bar{F}_{n}(u)=-N(u), \quad u>0 .
\end{aligned}
$$

The similarity between (2.2) and (2.4) is fundamental to our analysis. In the next section, it will be used for the Gumbel max domain of attraction and we will derive limit laws for power sums. As a second step, we then use the following lemma by Bogachev [2] to derive the limit distributions for the corresponding norms.

Lemma 2.1 ([2], Lemma 9.1) Let $\{S(t), t \geq 0\}$ be a family of positive random variables, such that for some (non-negative) functions $B(t), A(t)$ and a non-degenerate random variable $Y$,

$$
S^{*}(t):=\frac{S(t)-B(t)}{A(t)} \Rightarrow Y \quad(t \rightarrow \infty),
$$

where $\Rightarrow$ stands for convergence in distribution. Set $R(t):=S(t)^{1 / t}$ and $B^{*}(t):=$ $B(t) / A(t)$.

(a) If $B^{*}(t) \rightarrow \infty$ as $t \rightarrow \infty$, then

$$
t B^{*}(t)\left(\frac{R(t)}{B(t)^{1 / t}}-1\right) \Rightarrow Y \quad(t \rightarrow \infty) .
$$

(b) If $B^{*}(t) \equiv 0$ then

$$
t\left(\frac{R(t)}{A(t)^{1 / t}}-1\right) \Rightarrow \log Y \quad(t \rightarrow \infty) .
$$




\section{Main results for the Gumbel case}

Throughout this section let $X_{1}, X_{2}, \ldots$ be a sequence of i.i.d. positive random variables with distribution function $F \in \mathcal{D}_{\infty}(\Lambda)$. Then, according to (2.2), there exist norming constants $a(n)$ and $b(n)$, such that

$$
\lim _{n \rightarrow \infty} n \bar{F}(a(n) x+b(n))=-\log (\Lambda(x))=\exp (-x) .
$$

If the summands $\xi_{n, k}:=\left(X_{k}-b(n)\right) / a(n)$ were infinitesimal, Eq. (3.1) would ensure that the convergence criterion (2.4) is met for the sums $\sum_{i=1}^{n} \xi_{n, i}$. However, since

$$
\begin{aligned}
\sup _{1 \leq k \leq n} P\left(\left|\frac{X_{k}-b(n)}{a(n)}\right| \geq \epsilon\right) & \geq \sup _{1 \leq k \leq n} P\left(\frac{X_{k}-b(n)}{a(n)} \leq-\epsilon\right) \\
& \geq P\left(\frac{\max _{1 \leq k \leq n} X_{k}-b(n)}{a(n)} \leq-\epsilon\right) \\
& \rightarrow \Lambda(-\epsilon)>0, \quad n \rightarrow \infty,
\end{aligned}
$$

we know that this is not the case. To apply the connection between (2.2) and (2.4), we use a power transformation of the summands. It follows from the domain of the Gumbel distribution, which is $(-\infty, \infty)$, that

$$
b(n) / a(n) \rightarrow \infty
$$

as $n \rightarrow \infty$. Let us replace $x$ in (3.1) by

$$
g_{n}(x):=\frac{b(n)}{a(n)}\left(x^{a(n) / c b(n)}-1\right)
$$

with $c>0$. Because of (3.3) this function converges pointwise to $c^{-1} \log x$ as $n \rightarrow \infty$. Therefore,

$$
\begin{aligned}
n \bar{F}\left(b(n) x^{a(n) / c b(n)}\right) & =n \bar{F}\left(a(n) g_{n}(x)+b(n)\right) \\
& =n \bar{F}\left(a(n)\left(c^{-1} \log x+o(1)\right)+b(n)\right) \\
& \sim-\log \left(\Lambda\left(c^{-1} \log x\right)\right)=x^{-1 / c}, \quad n \rightarrow \infty
\end{aligned}
$$

where the asymptotic relation follows from the local uniformity of weak convergence to a continuous limit (see [5], p. 149). Note that formula (3.4) equals condition (2.4) for sums of the form

$$
\sum_{i=1}^{n}\left(\frac{X_{i}}{b(n)}\right)^{c b(n) / a(n)}
$$

which consist of infinitesimal summands. Consequently, we will analyze limit theorems for power sums of the form (3.5). An analogous procedure can be applied to the Weibull case, while the power transformation is not needed in the Fréchet case. 
For further investigation of the Gumbel case, the so-called von Mises representation of a distribution function $F \in \mathcal{D}_{\infty}(\Lambda)$ will be used.

Lemma 3.1 ([10], Proposition 1.4) A distribution function $F$ with upper endpoint $x_{\infty} \leq \infty$ belongs to the Gumbel max domain of attraction if and only if for $x \in$ $\left(-\infty, x_{\infty}\right)$ there exists a representation

$$
\bar{F}(x)=\bar{c}(x) \exp \left(-\int_{z_{0}}^{x} \frac{\mathbb{1}_{\left(z_{0}, x_{\infty}\right)}(u)}{f(u)} d u\right)
$$

for some $z_{0}<x_{\infty}$ with $\lim _{x \rightarrow x_{\infty}} \bar{c}(x)=\bar{c}>0$ and an absolutely continuous strictly positive function $f$ on $\left[z_{0}, x_{\infty}\right)$ with $\lim _{x \rightarrow x_{\infty}} f^{\prime}(x)=0$.

We are now able to formulate our main result.

Theorem 3.2 Let $X_{1}, X_{2}, \ldots$ be positive i.i.d. random variables with distribution function $F \in \mathcal{D}_{\infty}(\Lambda)$. Choose norming constants for the sequence of maxima

$$
b(n)=F^{\leftarrow}(1-1 / n) \text { and } a(n)=f(b(n))
$$

with $F^{\leftarrow}(x)=\inf \{t \in \mathbb{R}: F(t) \geq x\}$, f from the von Mises representation (3.6) and define $p(n):=b(n) / a(n)$. There exists a family $F_{c}$ of distribution functions of the form given below and a subsequence $n_{k} \rightarrow \infty$ such that for every $c>0$ there exist norming constants $\hat{a}_{c}\left(n_{k}\right), \hat{b}_{c}\left(n_{k}\right), k \in \mathbb{N}$, so that

$$
F_{c}(x)=\lim _{k \rightarrow \infty} P\left(\frac{\sum_{i=1}^{n_{k}} X_{i}^{c p\left(n_{k}\right)}-\hat{b}_{c}\left(n_{k}\right)}{\hat{a}_{c}\left(n_{k}\right)} \leq x\right) .
$$

The norming constants $\hat{a}_{c}\left(n_{k}\right)$ and $\hat{b}_{c}\left(n_{k}\right)$ can be chosen according to Table 1 .

Table 1 Norming constants

\begin{tabular}{lll}
\hline$c$ & $\hat{a}_{c}(n)$ & $\hat{b}_{c}(n)$ \\
\hline $0<c \leq \frac{1}{2}$ & $\sqrt{n \operatorname{Var}\left(X_{1}^{c p(n)} \mathbb{1}_{\left\{X_{1} \leq b(n)\right\}}\right.}$ & $n E\left(X_{1}^{c p(n)} \mathbb{1}_{\left\{X_{1}^{c p(n)} \leq \hat{a}_{c}(n)\right\}}\right)$ \\
$\frac{1}{2}<c<1$ & $b(n)^{c p(n)}$ & $n E\left(X_{1}^{c p(n)} \mathbb{1}_{\left\{X_{1} \leq b(n)\right\}}\right)$ \\
$c=1$ & $b(n)^{c p(n)}$ & $+\frac{b(n)^{c p(n)}}{1-c}$ \\
$c>1$ & $b(n)^{c p(n)}$ & $n E\left(X_{1}^{c p(n)} \mathbb{1}_{\left\{X_{1} \leq b(n)\right\}}\right)$ \\
\hline
\end{tabular}


If $0<c \leq \frac{1}{2}$, the $F_{c}$ equal the standard normal distribution function $N_{0,1}$.

If $\frac{1}{2}<c<\infty$, the $F_{c}$ are given by $F_{c}(x)=G_{1 / c}(x)$, where $G_{1 / c}$ is an $\alpha$-stable distribution function with $\alpha=1 / c$, skewness $\beta=1$ and characteristic function

$$
\phi_{1 / c}(u)= \begin{cases}\exp \left(-\Gamma(1-1 / c)|u|^{1 / c} \exp \left(-\frac{i \pi}{2 c} \operatorname{sgn} u\right)\right), & c \neq 1 \\ \exp \left(i u(1-\gamma)-\frac{\pi}{2}|u|\left(1+i \operatorname{sgn} u \frac{2}{\pi} \log u\right)\right), & c=1 .\end{cases}
$$

Here, $\gamma$ is the Euler constant, and $\Gamma(\cdot)$ is the gamma function.

The limit laws for $l_{p(n)}$-norms, which are missing in [11], are readily obtained with the help of Lemma 2.1.

Corollary 3.3 Let $X_{1 n}$ and $p(n)$ be as in Theorem 3.2. There exists a family $\tilde{F}_{c}$ of distribution functions of the form given below and a subsequence $n_{k} \rightarrow \infty$ such that for every $c>0$ there exist norming constants $\tilde{a}_{c}\left(n_{k}\right), \tilde{b}_{c}\left(n_{k}\right), k \in \mathbb{N}$, so that

$$
\tilde{F}_{c}(x)=\lim _{k \rightarrow \infty} P\left(\frac{\left\|X_{1 n_{k}}\right\|_{c p\left(n_{k}\right)}-\tilde{b}_{c}\left(n_{k}\right)}{\tilde{a}_{c}\left(n_{k}\right)} \leq x\right) .
$$

If $0<c \leq \frac{1}{2}$, the $\tilde{F}_{c}$ equal the standard normal distribution function $N_{0,1}$.

If $\frac{1}{2}<c \leq 1$, the $\tilde{F}_{c}$ are given by $\tilde{F}_{c}(x)=G_{1 / c}(x)$.

If $1<c<\infty$, the $\tilde{F}_{c}$ are given by $\tilde{F}_{c}(x)=G_{1 / c}(\exp (x))$.

Furthermore,

$$
\tilde{F}_{c}(c x) \rightarrow \Lambda(x), \quad c \rightarrow \infty .
$$

Proof The existence of norming constants and the form of the limit distributions follow from Lemma 2.1. We use part (a) of the lemma if $c \leq 1$ and part (b) if $c>1$. Relation (3.8) has been shown in [2], Theorem 10.2.

\section{Proof of Theorem 3.2}

\subsection{Main idea}

We use the abovementioned theorem for limit laws of sums (see [7], pp. 116-117). It has already been shown that the summands in (3.5) are infinitesimal. Let $F_{n}^{c}$ denote the distribution function of $\left(X_{1} / b(n)\right)^{c p(n)}$. Since $F_{n}^{c}(u)=0$ for all $u<0$, from (2.3) we readily obtain $M(\cdot) \equiv 0$. For $u>0$ it follows from (2.4) and (3.4) that for any subsequence $n_{k} \rightarrow \infty$,

$$
N(u)=-\lim _{k \rightarrow \infty} n_{k} \overline{F_{n_{k}}^{c}}(u)=-u^{-1 / c}, \quad u>0
$$


We are now left to prove (see [7], p. 116) that, along a certain subsequence $n_{k}$,

$$
\begin{aligned}
& \lim _{\epsilon \rightarrow 0} \liminf _{k \rightarrow \infty} n_{k}\left(\int_{0}^{\epsilon} x^{2} d F_{n_{k}}^{c}-\left(\int_{0}^{\epsilon} x d F_{n_{k}}^{c}(x)\right)^{2}\right) \\
& =\lim _{\epsilon \rightarrow 0} \limsup _{k \rightarrow \infty} n_{k}\left(\int_{0}^{\epsilon} x^{2} d F_{n_{k}}^{c}-\left(\int_{0}^{\epsilon} x d F_{n_{k}}^{c}(x)\right)^{2}\right)=: \sigma^{2} \in[0, \infty) .
\end{aligned}
$$

Note that

$$
0 \leq \int_{0}^{\varepsilon} x^{2} d F_{n_{k}}^{c}(x)-\left(\int_{0}^{\varepsilon} x d F_{n_{k}}^{c}(x)\right)^{2} \leq \int_{0}^{\varepsilon} x^{2} d F_{n_{k}}^{c}(x),
$$

and we will in fact show that, for an appropriate subsequence $n_{k}$,

$$
\lim _{k \rightarrow \infty} n_{k} \int_{0}^{\varepsilon} x^{p} d F_{n_{k}}^{c}(x)=\frac{1}{c p-1} \varepsilon^{p-1 / c}, \quad p>1 / c,
$$

which will readily imply that $\sigma^{2}=0$. The limit (4.3) would follow immediately if one could interchange limit and integration. We will choose a proper subsequence in order to justify this interchange by dominated convergence.

\subsection{Choice of a proper subsequence}

Let $f$ be given by (3.6). Choose a sequence $b_{k} \rightarrow x_{\infty}$ such that for the function $g(x):=f(x) / x$ the inequality $g(y) \geq g\left(b_{k}\right)$ holds for all $y \in\left(z_{0}, b_{k}\right)$ and all $k \in \mathbb{N}$. For instance, since $g$ is continuous and converges to zero (see [10], Lemma 1.2), set $b_{k}:=\min \left\{x \in\left[z_{0}, x_{\infty}\right) \mid g(x) \leq 1 / k\right\}$. Setting $n_{k}:=\left\lfloor 1 / \bar{F}\left(b_{k}\right)\right\rfloor$, where $\lfloor x\rfloor$ denotes the integer part of $x$, it follows that

$$
\begin{aligned}
n_{k} \bar{F}\left(f\left(b_{k}\right) x+b_{k}\right) & \sim \frac{\bar{F}\left(f\left(b_{k}\right) x+b_{k}\right)}{\bar{F}\left(b_{k}\right)} \\
& =\frac{\bar{c}\left(f\left(b_{k}\right) x+b_{k}\right)}{\bar{c}\left(b_{k}\right)} \exp \left(-\int_{b_{k}}^{f\left(b_{k}\right) x+b_{k}} \frac{\mathbb{1}_{\left(z_{0}, x_{\infty}\right)}(u)}{f(u)} d u\right) \\
& \sim \exp (-x), \quad k \rightarrow \infty,
\end{aligned}
$$

with the same argumentation as in the proof of Lemma 3.1 (see [10], p. 42). Therefore, both sequences $\left(f\left(b_{k}\right), b_{k}\right)$ and $\left(a\left(n_{k}\right), b\left(n_{k}\right)\right)$ can be used as norming constants 
for the maxima along the subsequence $n_{k}$. By the Convergence to Types Theorem, it follows that

$$
\lim _{k \rightarrow \infty} \frac{a\left(n_{k}\right)}{f\left(b_{k}\right)}=\lim _{k \rightarrow \infty} \frac{f\left(b\left(n_{k}\right)\right)}{f\left(b_{k}\right)}=1 \text { and } \lim _{k \rightarrow \infty} \frac{b_{k}-b\left(n_{k}\right)}{f\left(b_{k}\right)}=0 .
$$

In the following let $n_{k}$ be chosen as described above.

\subsection{Application of dominated convergence}

Partial integration in (4.3) yields

$$
\begin{aligned}
n_{k} \int_{0}^{\varepsilon} x^{p} d F_{n_{k}}^{c}(x) & =-n_{k} \int_{0}^{\varepsilon} x^{p} d \overline{F_{n_{k}}^{c}}(x) \\
& =-n_{k} \varepsilon^{p} \overline{F_{n_{k}}^{c}}(\varepsilon)+n_{k} p \int_{0}^{\varepsilon} x^{p-1} \overline{F_{n_{k}}^{c}}(x) d x,
\end{aligned}
$$

where the first term in (4.6) converges to $-\varepsilon^{p-1 / c}$ for every subsequence $n_{k} \rightarrow \infty$ by (3.4). In what follows let $\beta_{k}^{c}:=\frac{f\left(b\left(n_{k}\right)\right)}{c b\left(n_{k}\right)}$. Now,

$$
\begin{aligned}
n_{k} \overline{F_{n_{k}}^{c}}(x)= & n_{k} \bar{F}\left(b\left(n_{k}\right) x^{\beta_{k}^{c}}\right) \\
\sim & \frac{\bar{F}\left(b\left(n_{k}\right) x^{\beta_{k}^{c}}\right)}{\bar{F}\left(b\left(n_{k}\right)\right)} \\
= & \frac{\bar{c}\left(b\left(n_{k}\right) x^{\beta_{k}^{c}}\right)}{\bar{c}\left(b\left(n_{k}\right)\right)} \exp \left(\int_{b\left(n_{k}\right) x^{\beta_{k}^{c}}}^{b\left(n_{k}\right)} \frac{\mathbb{1}_{\left(z_{0}, x_{\infty}\right)}(u)}{f(u)} d u\right) \\
= & \frac{\bar{c}\left(b\left(n_{k}\right) x^{\beta_{k}^{c}}\right)}{\bar{c}\left(b\left(n_{k}\right)\right)} \exp \left(\frac{1}{c} \int_{\log x}^{0} \frac{f\left(b\left(n_{k}\right)\right) \exp \left(\beta_{k}^{c} u\right)}{f\left(b\left(n_{k}\right) \exp \left(\beta_{k}^{c} u\right)\right)}\right. \\
& \left.\times \mathbb{1}_{\left(z_{0}, x_{\infty}\right)}\left(b\left(n_{k}\right) \exp \left(\beta_{k}^{c} u\right)\right) d u\right) .
\end{aligned}
$$

With the previously defined $g$ we get

$$
n_{k} \overline{F_{n_{k}}^{c}}(x) \sim \frac{\bar{c}\left(b\left(n_{k}\right) x^{\beta_{k}^{c}}\right)}{\bar{c}\left(b\left(n_{k}\right)\right)} \exp \left(\frac{1}{c} \int_{\log x}^{0} \frac{g\left(b\left(n_{k}\right)\right)}{g\left(b\left(n_{k}\right) \exp \left(\beta_{k}^{c} u\right)\right)} \mathbb{1}_{\left(z_{0}, x_{\infty}\right)}\left(b\left(n_{k}\right) \exp \left(\beta_{k}^{c} u\right)\right) d u\right) .
$$


For $\epsilon \in(0, \bar{c})$ there exists a $k(\epsilon) \in \mathbb{N}$ such that $\left|\bar{c}\left(b\left(n_{k}\right)\right)-\bar{c}\right|<\epsilon$ for all $k>k(\epsilon)$. Since $\bar{F}$ lies between 0 and 1 and the exponential part of $\bar{F}$ in (3.6) is monotonically decreasing, it follows that

$$
\bar{c}(y) \leq \exp \left(\int_{z_{0}}^{b\left(n_{k(\epsilon)}\right)} \frac{\mathbb{1}_{\left(z_{0}, x_{\infty}\right)}(u)}{f(u)} d u\right)
$$

for $y<b\left(n_{k(\epsilon)}\right)$. Therefore, we can find a constant $C>0$ such that

$$
\frac{\bar{c}\left(b\left(n_{k}\right) x^{\beta_{k}^{c}}\right)}{\bar{c}\left(b\left(n_{k}\right)\right)} \leq C
$$

for all $x<1$ if $k$ is large enough. For such $x$ and $k$ we get

$$
\begin{aligned}
n_{k} \overline{F_{n_{k}}^{c}}(x) & \leq C \exp \left(\frac{1}{c} \int_{\log x}^{0} \frac{g\left(b_{k}\right)(1+\epsilon)}{g\left(b\left(n_{k}\right) \exp \left(\beta_{k}^{c} u\right)\right)} \mathbb{1}_{\left(z_{0}, x_{\infty}\right)}\left(b\left(n_{k}\right) \exp \left(\beta_{k}^{c} u\right)\right) d u\right) \\
& \leq C \exp \left(-\frac{1+\epsilon}{c} \log x\right)
\end{aligned}
$$

because of (4.5), the choice of $n_{k}$ and since $b\left(n_{k}\right) \leq b_{k}$.

Taking the limit in (4.6), we can interchange limit and integral by Lebesgue's Theorem to obtain

$$
\begin{aligned}
\lim _{k \rightarrow \infty} n_{k} \int_{0}^{\varepsilon} x^{p} d F_{n_{k}}^{c}(x) & =-\varepsilon^{p-1 / c}+p \int_{0}^{\varepsilon} x^{p-1} x^{-1 / c} d x \\
& =-\varepsilon^{p-1 / c}+\frac{c p}{c p-1} \varepsilon^{p-1 / c}=\frac{1}{c p-1} \varepsilon^{p-1 / c} .
\end{aligned}
$$

4.4 Limit distributions and norming constants for $c>\frac{1}{2}$

For $c>\frac{1}{2}$ both (2.4) and (4.2) are met and the limit law has characteristic function $\phi(\cdot)$, where

$$
\log (\phi(t))=i \mu t-\frac{\sigma^{2}}{2} t^{2}+\int_{|u|>0}\left(e^{i u t}-1-\frac{i u t}{1+u^{2}}\right) d N(u)
$$

(see [7], p. 117), with

$$
N(u)=-u^{-1 / c}, \quad \sigma^{2}=0,
$$


and location parameter $\mu$ yet to be determined. Hence, the limit law is $\alpha$-stable (see [7], p. 164) with $\alpha=1 / c$ and skewness parameter $\beta=1$. The value of $\mu$ depends on the choice of additive norming constants. We choose

$$
B_{c}(n)= \begin{cases}0 & \text { for } c>1 \\ n E\left(X_{1}^{c p(n)} \mathbb{1}_{\left\{X_{1} \leq b(n)\right\}}\right) & \text { for } c=1 \\ n E\left(X_{1}^{c p(n)} \mathbb{1}_{\left\{X_{1} \leq b(n)\right\}}\right)+\frac{b(n)^{c p(n)}}{1-c} & \text { for } 1 / 2<c<1 .\end{cases}
$$

Then, for $\tau>0$, it follows that (see [7], p. 84, 117)

$$
\begin{aligned}
\mu= & \lim _{k \rightarrow \infty}\left[n _ { k } E \left(\left(\frac{X_{1}}{b\left(n_{k}\right)}\right)^{c p\left(n_{k}\right)} \mathbb{1}_{\left.\left.\left\{\left(\frac{X_{1}}{b\left(n_{k}\right)}\right)^{c p\left(n_{k}\right)} \leq \tau\right\}\right)-\frac{B_{c}\left(n_{k}\right)}{b\left(n_{k}\right)^{c p\left(n_{k}\right)}}\right]}\right.\right. \\
& -\int_{0}^{\tau} \frac{x^{3}}{1+x^{2}} d N(x)+\int_{\tau}^{\infty} \frac{x}{1+x^{2}} d N(x) .
\end{aligned}
$$

Using (4.9) and considering various cases for the constant $c$ we obtain

- For $c>1$ :

$$
\begin{aligned}
\mu & =\lim _{k \rightarrow \infty} n_{k} \int_{0}^{\tau} x d F_{n_{k}}^{c}(x)-0-\frac{1}{c} \int_{0}^{\tau} \frac{x^{2-1 / c}}{1+x^{2}} d x+\frac{1}{c} \int_{\tau}^{\infty} \frac{x^{-1 / c}}{1+x^{2}} d x \\
& =\frac{1}{c} \int_{0}^{\tau}\left(x^{-1 / c}-\frac{x^{2-1 / c}}{1+x^{2}}\right) d x+\frac{1}{c} \int_{\tau}^{\infty} \frac{x^{-1 / c}}{1+x^{2}} d x \\
& =\frac{1}{c} \int_{0}^{\infty} \frac{x^{-1 / c}}{1+x^{2}}=\frac{\pi}{2 c \cos \left(\frac{\pi}{2 c}\right)}
\end{aligned}
$$

by (4.3) and [8], \# 3.241(2).

- For $c=1$ :

$$
\begin{aligned}
\mu= & \lim _{k \rightarrow \infty} n_{k}\left(\int_{0}^{\tau} x d F_{n_{k}}^{c}(x)-\int_{0}^{1} x d F_{n_{k}}^{c}(x)\right) \\
& -\frac{1}{c} \int_{0}^{\tau} \frac{x}{1+x^{2}} d x+\frac{1}{c} \int_{\tau}^{\infty} \frac{x^{-1}}{1+x^{2}} d x \\
= & \frac{1}{c} \int_{1}^{\tau} x^{-1} d x-\frac{1}{c} \int_{1 / \tau}^{\tau} \frac{x}{1+x^{2}} d x=\frac{1}{c} \log \tau-\frac{1}{c} \log \tau=0,
\end{aligned}
$$


since limit and integration are interchangeable on the interval $(1, \tau)$, and because of [8], \# 2.145(2).

- For $1 / 2<c<1$ :

$$
\begin{aligned}
\mu= & \lim _{k \rightarrow \infty} n_{k}\left(\int_{0}^{\tau} x d F_{n_{k}}^{c}(x)-\int_{0}^{1} x d F_{n_{k}}^{c}(x)\right)-\frac{1}{1-c} \\
& -\frac{1}{c} \int_{0}^{\tau} \frac{x^{2-1 / c}}{1+x^{2}} d x+\frac{1}{c} \int_{\tau}^{\infty} \frac{x^{-1 / c}}{1+x^{2}} d x \\
= & -\frac{1}{c} \int_{\tau}^{\infty}\left(x^{-1 / c}-\frac{x^{-1 / c}}{1+x^{2}}\right) d x-\frac{1}{c} \int_{0}^{\tau} \frac{x^{2-1 / c}}{1+x^{2}} d x \\
= & -\frac{1}{c} \int_{0}^{\infty} \frac{x^{2-1 / c}}{1+x^{2}} d x=\frac{\pi}{2 c \cos \left(\frac{\pi}{2 c}\right)}
\end{aligned}
$$

again by (4.3) and [8], \# 3.241(2).

It is shown in [1], Theorem 6.2, that the characteristic functions (4.8) may be written in the form (3.7) with constants as stated in Theorem 3.2.

\subsection{Limit distributions and norming constants for $c \leq \frac{1}{2}$}

The case $c \leq \frac{1}{2}$ is studied seperately, as we have to change the multiplicative norming constants to keep the sum of the truncated variances bounded. According to [7], pp. 130-131, for a given subsequence $n_{k}$, it suffices to find a sequence $C_{n_{k}} \rightarrow \infty$ such that

$$
\lim _{k \rightarrow \infty} n_{k} \int_{|x|>C_{n_{k}}} d F_{n_{k}}^{c}=0
$$

and

$$
\lim _{k \rightarrow \infty} \frac{n_{k}}{C_{n_{k}}^{2}}\left(\int_{|x|<C_{n_{k}}} x^{2} d F_{n_{k}}^{c}(x)-\left(\int_{|x|<C_{n_{k}}} x d F_{n_{k}}^{c}(x)\right)^{2}\right)=\infty
$$

To satisfy condition (4.11), we subtract the median $m_{n}$ from each variable (3.5) as done in [6]. It follows from (3.4) that $m_{n}$ tends to zero. From local uniform convergence we obtain

$$
n_{k} \overline{F_{n_{k}}^{c, m}}(x) \sim x^{-1 / c}, \quad x>0,
$$


where $F_{n_{k}}^{c, m}$ denotes the distribution function of the median-subtracted variables. Hence, (4.10) is valid for every sequence $C_{n_{k}}$ that tends to infinity. To verify (4.11) we make use of the median-normalization. Feller shows (see [6], p. 527) that it suffices to find a sequence $C_{n_{k}}$ such that

$$
\lim _{k \rightarrow \infty} \frac{n_{k}}{C_{n_{k}}^{2}}\left(\int_{|x|<C_{n_{k}}} x^{2} d F_{n_{k}}^{c, m}(x)\right)=\infty .
$$

But it follows from (4.12) and Fatou's Lemma that

$$
\lim _{k \rightarrow \infty} n_{k} \int_{|x|<\epsilon} x^{2} d F_{n_{k}}^{c, m}(x)=\infty
$$

for any $\epsilon>0$. Hence, an appropriate sequence $C_{n_{k}}$ can be constructed. We can choose norming constants as given in the theorem.

\section{Further results for the Gumbel case}

The following corollary shows that in some cases the restriction to certain subsequences is not necessary.

Corollary 5.1 Let $X_{1}, X_{2}, \ldots$ be i.i.d. with distribution function $F \in \mathcal{D}_{\infty}(\Lambda)$ with representation (3.6) and let $g(x):=f(x) / x$ be ultimately monotone. Let $p(n)$ be defined as in Theorem 3.2. Then, for every $c>0$, there exist norming constants $\hat{a}_{c}(n), \hat{b}_{c}(n)$ such that

$$
\lim _{n \rightarrow \infty} P\left(\frac{\sum_{i=1}^{n} X_{i}^{c p(n)}-\hat{b}_{c}(n)}{\hat{a}_{c}(n)} \leq x\right)=F_{c}(x),
$$

with $F_{c}$ defined as in Theorem 3.2. The norming constants may be chosen according to Table 1.

Proof If $g$ is ultimately monotone, the constant $z_{0}$ can be shifted in such a way that $g$ is monotonically decreasing on $\left(z_{0}, x_{\infty}\right)$. The proof of Theorem 3.2 then shows the convergence for the whole sequence.

The following example illustrates that the restriction in Theorem 3.2 is not purely technical.

Example 5.2 Let $X_{1}, X_{2}, \ldots$ be i.i.d. with tail distribution function

$$
\bar{F}(x)=\exp \left(-\int_{0}^{x} \frac{u}{1.5+\sin (u)} d u\right) .
$$


Then $F \in \mathcal{D}_{\infty}(\Lambda)$, but there exist $c>0$ and a sequence $n_{k} \rightarrow \infty$ such that no norming constants exist that ensure convergence of the power sums $\sum_{i=1}^{n_{k}} X_{i}^{c p\left(n_{k}\right)}$ to the limit distribution function $F_{c}$ of Theorem 3.2.

Proof Set $f(x)=x^{-1}(1.5+\sin (x))$. Since $f^{\prime}(x) \rightarrow 0$ as $x \rightarrow x_{\infty}=\infty$, it follows from Lemma 3.1 that $F$ has a von Mises representation (3.6), and therefore $F \in \mathcal{D}_{\infty}(\Lambda)$. Now, let us choose a subsequence that does not allow for the interchange of limit and integral on the left hand side of (4.3). Here, the idea is to find a sequence $n_{k}$ such that the integrand in (4.7) is large for small values of $x$. By reasoning similar to the proof of Theorem 3.2, it is possible to choose a subsequence $n_{k}$ such that $b\left(n_{k}\right)=(k+0.5) \pi$ are suitable norming constants for the maxima. With $g(x)=f(x) / x=x^{-2}(1.5+\sin (x))$, along this sequence we have $g\left(b\left(n_{k}\right)\right)=2.5((k+0.5) \pi)^{-2}$. It follows for the second term on the right hand side of (4.6) that

$$
\begin{aligned}
2 n_{k} \int_{0}^{\varepsilon} x \overline{F_{n_{k}}^{c}}(x) d x & \sim 2 \int_{0}^{\varepsilon} \exp \left(\log x+\int_{b\left(n_{k}\right) x^{\beta_{k}^{c}}}^{b\left(n_{k}\right)} \frac{1}{f(u)} d u\right) d x \\
& =2 \int_{-\infty}^{\log \varepsilon} \exp \left(2 y+\int_{b\left(n_{k}\right) \exp \left(\beta_{k}^{c} y\right)}^{b\left(n_{k}\right)} \frac{1}{f(u)} d u\right) d y .
\end{aligned}
$$

Next, we will show that $-(2+\epsilon) y$ is a lower bound for the inner integral in (5.1) in certain regions of the integration range. To this end, we analyze the behavior of the inner integral for $y$ in a neighborhood of

$$
y_{k}:=\log \left(\frac{k-0.5}{k+0.5}\right) \frac{(k+0.5)^{2} \pi^{2}}{2.5}=-\frac{(k+0.5) \pi^{2}}{2.5}+o(1) .
$$

Choose $\delta>0$ and $y \in\left(y_{k}-\delta, y_{k}+\delta\right)$ with $\delta(y):=y-y_{k}$. It follows that

$$
\begin{aligned}
& -\frac{1}{y} \int_{b\left(n_{k}\right) \exp \left(\left(y_{k}+\delta(y)\right) \beta_{k}^{c}\right)}^{b\left(n_{k}\right)} \frac{1}{f(u)} d u \\
& =\int_{(k-0.5) \pi \exp \left(\delta(y) g\left(b\left(n_{k}\right)\right) / c\right)}^{(k+0.5) \pi} \frac{u}{1.5+\sin (u)} d u \frac{2.5}{(k+0.5) \pi^{2}}(1+o(1)) \\
& =\int_{(k-0.5) \pi}^{(k+5) \pi+o(1)} \frac{1}{1.5+\sin (u)} d u\left(\frac{2.5}{\pi}+o(1)\right)
\end{aligned}
$$




$$
\begin{aligned}
= & \int_{-0.5 \pi+o(1)}^{0.5 \pi} \frac{1}{1.5+\sin (u)} d u\left(\frac{2.5}{\pi}+o(1)\right) \\
= & \frac{2}{\sqrt{1.25}}\left(\arctan \left(\frac{1.5 \tan (\pi / 4)+1}{\sqrt{1.25}}\right)-\arctan \left(\frac{1.5 \tan (-\pi / 4)+1}{\sqrt{1.25}}\right)\right) \\
& \times\left(\frac{2.5}{\pi}+o(1)\right) \approx 2.236+o(1),
\end{aligned}
$$

by use of [8], \#2.551(3).

Hence, for every $\epsilon>0$ and $\delta>0$ there exists a $k(\delta, \epsilon) \in \mathbb{N}$ such that

$$
\int_{b\left(n_{k}\right) \exp \left(y \frac{a\left(n_{k}\right)}{c b\left(n_{k}\right)}\right)}^{b\left(n_{k}\right)} \frac{1}{f(u)} d u \geq-(2.2-\epsilon) y
$$

for all $y \in\left(y_{k}-\delta, y_{k}+\delta\right)$ with $k>k(\delta, \epsilon)$. The integrand in (5.1) is therefore bounded from below by $\exp (-(0.2-\epsilon) y)$ in a region of length $2 \delta$ that tends to $-\infty$ as $k \rightarrow \infty$. This prevents the convergence of the integral on the right hand side of (4.3) as $k \rightarrow \infty$. Now, since we have shown that the lim sup in (4.2) is infinite, we can argue similarly to the case $c \leq 1 / 2$ in the proof of Theorem 3.2. Namely, the distributions of the power sums converge to a normal distribution along this subsequence. By the Convergence to Types Theorem this prevents convergence to $F_{c}$ for $c>1 / 2$.

Note the analogy of this example to distributions that have a rather light tail but are not in $\mathcal{D}_{\infty}(\Lambda)$ because of their discreteness (e.g., a binomial distribution). Similar to discrete laws, the tail distribution function given in the example above has a somewhat stairlike appearance since $f$ is an oscillating function.

The next theorem shows that the limit distributions that are not covered by Theorem 3.2 are quite similar to the ones studied so far.

Theorem 5.3 Let $X_{1 n}$ and $p(n)$ be defined as in Theorem 3.2. Assume that there exist $c>0$, a subsequence $n_{k} \rightarrow \infty$, and norming constants $\hat{a}_{c}\left(n_{k}\right)$ and $\hat{b}_{c}\left(n_{k}\right)$ such that a limit distribution function

$$
\hat{F}_{c}(x)=\lim _{k \rightarrow \infty} P\left(\frac{\sum_{i=1}^{n_{k}} X_{i}^{c p\left(n_{k}\right)}-\hat{b}_{c}\left(n_{k}\right)}{\hat{a}_{c}\left(n_{k}\right)} \leq x\right)
$$

exists and is not of the same type as the $F_{c}$ in Theorem 3.2, i.e., there exists no linear transformation $l(\cdot)$ such that $\hat{F}_{c}(x)=F_{c}(l(x))$ for all $x \in \mathbb{R}$. Then, $c>1 / 2$ and $\hat{F}_{c}$ is of the same type as $N_{0,1}$ or $G_{1 / c} \star N_{0, \sigma^{2}}$, where $\star$ denotes the convolution of distribution functions.

Proof Every limit law has to be infinitely divisible and is uniquely determined by its Lévy measure and $\sigma^{2}$. The Lévy measure is defined by $M(\cdot)$ and $N(\cdot)$ in (2.3) and (2.4). If we choose multiplicative norming constants as in (3.5), cf. the proof of 
Theorem 3.2, we have $M(\cdot) \equiv 0$, and $N(\cdot)$ of the form (4.1). With asymptotically different norming constants we obtain $M(\cdot) \equiv N(\cdot) \equiv 0$. Apart from the arbitrary location and scale parameters the limiting law is determined by whether it has a normal component (i.e. $\sigma^{2}>0$ in (4.8)) or not. To determine this component, consider the lim sup in (4.2). If it is infinite, then, similarly to the case $c \leq 1 / 2$ in the proof of Theorem 3.2, we obtain convergence to a normal distribution along a certain subsubsequence; if it equals zero, we obtain convergence to an $\alpha$-stable distribution; if it is finite and positive, by choosing the corresponding subsubsequence we obtain convergence to an $\alpha$-stable distribution convoluted with a normal distribution. Hence, the subsequence converges to a distribution of the stated form.

\section{The Weibull and Fréchet cases}

The limit laws of norms for the Weibull and the Fréchet max domain of attraction are studied in [11]. With the technique stated above we find limit distributions for power sums and are able to simplify some of the proofs in [11].

Let $X_{1}, X_{2}, \ldots$ be i.i.d. positive random variables with distribution function $F \in$ $\mathcal{D}_{\infty}\left(\Psi_{\alpha}\right)$ and upper endpoint $x_{\infty}<\infty$. Without loss of generality, $x_{\infty}=1$. Now, possible norming constants for the sequence of maxima are given by $a(n)=1-F^{\leftarrow}(1-$ $1 / n)$ and $b(n) \equiv 1$ (see [5], Theorem 3.3.12). Similar to (3.2) we can conclude that the summands $\xi_{n, k}:=\left(X_{k}-a(n)\right) / b(n)$ are not infinitesimal. Since $b(n) / a(n) \rightarrow \infty$ as $n \rightarrow \infty$, a power transformation allows us to analyze sums of the form (3.5). We obtain $M(\cdot) \equiv 0$ and

$$
N(u)=-\lim _{n \rightarrow \infty} n \overline{F_{n}^{c}}(u)=\log \left(\Psi_{\alpha}\left(\log \left(u^{1 / c}\right)\right)\right)=-\left(-\frac{\log u}{c}\right)^{\alpha}, \quad u \in(0,1),
$$

for the Lévy measure of the limit law of the power sums (3.5). To show that condition (4.2) is met we make use of a special representation of $F$ :

$$
\bar{F}(x)=\bar{c}(x) \exp \left(-\int_{0}^{x} \frac{\delta(t)}{1-t} d t\right)
$$

for $x \in(0,1)$ with $\delta: \mathbb{R}^{+} \rightarrow \mathbb{R}^{+}, \delta(t) \rightarrow \alpha$ and $\bar{c}(t) \rightarrow \bar{c}>0$ as $t \rightarrow 1$ (see [10], Corollary 1.14).

Equation (4.2) is again used to derive $\sigma^{2}$. Interchanging limit and integral in

$$
\lim _{n \rightarrow \infty} n p \int_{0}^{\epsilon} x^{p-1} \overline{F_{n}^{c}}(x) d x, \quad p>0
$$


is allowed because of dominated convergence: Choose the functions $\bar{c}$ and $\delta$ in (6.1) such that $|\delta(t)-\alpha|<\varepsilon$ for all $t \in(0,1)$ and some $\varepsilon$ with $0<\varepsilon<\alpha$. Now, we obtain

$$
\begin{aligned}
n \overline{F_{n}^{c}}(x) \sim & \frac{\bar{c}\left(x^{1 / c p(n)}\right)}{\bar{c}\left(F^{\leftarrow}(1-1 / n)\right)} \exp \left(-\int_{F^{\leftarrow}(1-1 / n)}^{x^{1 / c p(n)}} \frac{\delta(t)}{1-t} d t\right) \\
\leq & C \max \left[\exp \left(-(\alpha-\varepsilon) \int_{F^{\leftarrow}(1-1 / n)}^{x^{1 / c p(n)}} \frac{1}{1-t} d t\right),\right. \\
x^{1 / c p(n)} & \left.\times \exp \left(-(\alpha+\varepsilon) \quad \int_{F \leftarrow(1-1 / n)}^{1-t} d t\right)\right] \\
= & C \max \left\{\exp \left((\alpha-\varepsilon) \log \left(p(n)\left(1-x^{1 / c p(n)}\right)\right)\right),\right. \\
& \left.\quad \times \exp \left((\alpha+\varepsilon) \log \left(p(n)\left(1-x^{1 / c p(n)}\right)\right)\right)\right\} \\
\leq & C \max \left\{\left(-\log \left(x^{1 / c}\right)\right)^{\alpha-\varepsilon},\left(-\log \left(x^{1 / c}\right)\right)^{\alpha+\varepsilon}\right\} .
\end{aligned}
$$

It follows from (4.2) that $\sigma^{2}=0$.

Theorem 6.1 Let $X_{1}, X_{2}, \ldots$ be positive i.i.d. random variables with distribution function $F \in \mathcal{D}_{\infty}\left(\Psi_{\alpha}\right), \alpha>0$, and upper endpoint $0<x_{\infty}<\infty$. Let

$$
b(n) \equiv x_{\infty}, \quad a(n)=x_{\infty}-F^{\leftarrow}(1-1 / n) \quad \text { and } \quad p(n)=b(n) / a(n) .
$$

Then, there exists a family $F_{c}$ of distribution functions of the form given below such that for every $c>0$ there exist norming constants $\hat{a}_{c}(n), \hat{b}_{c}(n), n \in \mathbb{N}$, so that

$$
F_{c}(x)=\lim _{n \rightarrow \infty} P\left(\frac{\sum_{i=1}^{n} X_{i}^{c p(n)}-\hat{b}_{c}(n)}{\hat{a}_{c}(n)} \leq x\right) .
$$

The limit distribution functions are of the type $F_{c}(x)=H_{c}(x)$, where $H_{c}$ has characteristic function of the form (4.8) with $\sigma^{2}=0$ and $N(u)=-c^{-\alpha}(-\log u)^{\alpha} \mathbb{1}_{(0,1)}(u)$.

Corollary 6.2 ([11], Theorem 2.2) Let $X_{1 n}$ and $p(n)$ be as in Theorem 6.1. Then, for every $c>0$, there exist norming constants $\tilde{a}_{c}(n), \tilde{b}_{c}(n), n \in \mathbb{N}$, such that

$$
\tilde{H}_{c}(\exp (x))=\lim _{n \rightarrow \infty} P\left(\frac{\left\|X_{1 n}\right\|_{c p(n)}-\tilde{b}_{c}(n)}{\tilde{a}_{c}(n)} \leq x\right) .
$$

Let us conclude by considering the Fréchet max domain of attraction. In contrast to the cases studied above, a purely multiplicative normalization is sufficient for the sequences of maxima, i.e. $a(n)=F^{\leftarrow}(1-1 / n)$ and $b(n) \equiv 0$ (see [5], Theorem 
3.3.7). Since $a(n) \rightarrow \infty$ as $n \rightarrow \infty$, the variables $\xi_{n, k}=\left(X_{k}-b(n)\right) / a(n)$ are infinitesimal, here.

Let $X_{1}$ be a random variable with distribution function $F \in \mathcal{D}_{\infty}\left(\Phi_{\alpha}\right)$. Then, the random variable $X_{1}^{c}, c>0$, belongs to the sum domain of attraction of an $\alpha$-stable distribution (see, e.g., [5], Theorem 2.2.8). Hence, there exist norming constants $\hat{a}_{c}(n)$ and $\hat{b}_{c}(n)$ and a limit distribution function $F_{c}$ such that

$$
F_{c}(x)=\lim _{n \rightarrow \infty} P\left(\frac{\sum_{i=1}^{n} X_{i}^{c}-\hat{b}_{c}(n)}{\hat{a}_{c}(n)} \leq x\right)
$$

for all $x \in \mathbb{R}$. If $c<\alpha / 2$, the $F_{c}$ equal the normal distribution function $N_{0,1}$, whereas for $c \geq \alpha / 2$ they equal an $\alpha$-stable distribution function $G_{\alpha / c}$. This connection between limit laws for sums and for maxima of random variables with regularly varying tails is in fact well known and has been studied in [4]. Schlather's result for the limit laws of norms is stated below for completeness.

Corollary 6.3 ([11], Theorem 2.3) Let $X_{1 n}=\left(X_{1}, X_{2}, \ldots, X_{n}\right)$ where $X_{1}, X_{2}, \ldots$ are positive i.i.d. random variables with distribution function $F \in \mathcal{D}_{\infty}\left(\Phi_{\alpha}\right), \alpha>0$. There exists a family $\tilde{F}_{c}$ of distribution functions of the form given below such that for every $c>0$ there exist norming constants $\tilde{a}_{c}(n), \tilde{b}_{c}(n), n \in \mathbb{N}$, so that

$$
\tilde{F}_{c}(x)=\lim _{n \rightarrow \infty} P\left(\frac{\left\|X_{1 n}\right\|_{c}-\tilde{b}_{c}(n)}{\tilde{a}_{c}(n)} \leq x\right) .
$$

If $0<c \leq \frac{\alpha}{2}$, the $\tilde{F}_{c}$ equal the standard normal distribution function $N_{0,1}$.

If $\frac{\alpha}{2}<c \leq \alpha$, the $\tilde{F}_{c}$ are given by $\tilde{F}_{c}(x)=G_{\alpha / c}(c x)$.

If $\alpha<c<\infty$, the $\tilde{F}_{c}$ are given by $\tilde{F}_{c}(x)=G_{\alpha / c}\left(x^{c}\right)$.

Note the similarity between the family of laws that we obtained for the Gumbel case and the family for the Fréchet case with $\alpha=1$. For $c \leq 1$ the limit distribution functions $\tilde{F}_{c}$ are equal for the two cases whereas for $c>1$ the limit random variables in the Gumbel case are logarithms of the limit random variables in the Fréchet case. For $c \rightarrow \infty$ this reflects the relation $\Lambda=\Phi_{1} \circ \exp$.

Acknowledgments The author is grateful to Martin Schlather and Zakhar Kabluchko for hints and helpful discussions and would like to thank an anonymous referee for very valuable comments.

Open Access This article is distributed under the terms of the Creative Commons Attribution Noncommercial License which permits any noncommercial use, distribution, and reproduction in any medium, provided the original author(s) and source are credited.

\section{References}

1. Ben Arous, G., Bogachev, L.V., Molchanov, S.A.: Limit theorems for sums of random exponentials. Probab. Theory Relat. Fields. 132, 579-612 (2005)

2. Bogachev, L.: Limit laws for norms of IID samples with Weibull tails. J. Theor. Probab. 19, 849873 (2006) 
3. Bovier, A., Kurkova, I., Löwe, M.: Fluctuations of the free energy in the REM and the $p$-spin SK model. Ann. Probab. 30, 605-651 (2002)

4. Darling, D.A.: The influence of the maximum term in the addition of independent random variables. Trans. Am. Math. Soc. 73, 95-107 (1952)

5. Embrechts, P., Klüppelberg, C., Mikosch, T.: Modelling Extremal Events. Springer, Berlin (1997)

6. Feller, W.: Über den zentralen Grenzwertsatz der Wahrscheinlichkeitsrechnung. Math. Z. 40, 521559 (1936)

7. Gnedenko, B.V., Kolmogorov, A.N.: Limit Distributions for Sums of Independent Random Variables. Addison-Wesley, Reading (1968)

8. Gradshteyn, I.S., Ryzhik, I.M.: Table of integrals, series, and products, 6th edn. In: Jeffrey, A. (ed.) Academic Press, San Diego (2000)

9. Loève, M.: Probability Theory. Van Nostrand, New York (1955)

10. Resnick, S.I.: Extreme Values, Regular Variation and Point Processes. Springer, New York (1987)

11. Schlather, M.: Limit distributions of norms of vectors of positive i.i.d. random variables. Ann. Probab. 29, 862-881 (2001) 